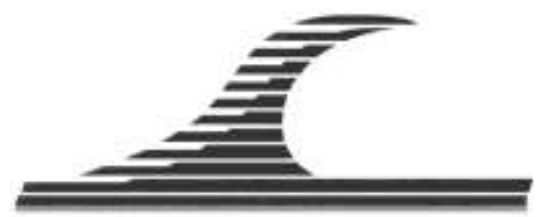

Revue Paralia, Volume 4 (2011) pp 9.1-9.10

Mots-clés : Ouvrage, Aménagement côtier, Dynamique

littorale, Modélisation numérique, Hydrodynamique,

Transport sédimentaire

(C) Editions Paralia CFL

\title{
Impact de digues ISC sur la dynamique littorale : Etude de cas des plages de Saint-Hilaire de Riez en Vendée
}

\author{
Julie LEBUNETEL ${ }^{1}$, Pierre FARNOLE ${ }^{1}$
}

1. ERAMM, 1503 rte des Dolines, BP 42, 06901 Sophia Antipolis, France.

jl@eramm.fr;pf@eramm.fr

\section{Résumé :}

Le littoral vendéen est exposé à des conditions de houle sévères et a subi ces dernières années d'importants désordres hydrosédimentaires. Les plages du littoral de SaintHilaire de Riez sont constituées d'une succession discontinue d'ouvrages de protection contre la mer sur un linéaire naturel. Ces perrés et digues en enrochements ou maçonnés ont été installés sans étude ciblée au préalable concernant leur impact sur leur environnement. Ces digues sont maintenant classées ISC (Intéressant la Sécurité Civile) et font l'objet d'un suivi particulier. Les dégradations importantes de ces ouvrages subies ces dernières années justifient la réalisation d'une étude hydrosédimentaire pour vérifier leur impact sur la dynamique littorale et proposer des solutions d'aménagement permettant de rétablir un équilibre sur ce littoral. Une chaîne de calcul de modélisation hydrosédimentaire, dont les logiciels sont entièrement développés par le CETMEF, a été mise en place. Cette étude vise à valider numériquement la stratégie de recul des ouvrages préconisée pour rétablir l'équilibre de la dynamique littorale de Saint-Hilaire de Riez.

Soumis le 29 octobre 2010, accepté le 8 juillet 2011, en ligne le 29 décembre 2011.

La seule version examinée est celle écrite en français. La ou les autres versions n'étant pas examinées par le comité de rédaction de la revue, sont donc publiées sous l'entière responsabilité du ou des auteurs.

A TRANSLATED VERSION IN ENGLISH IS AVAILABLE ONLINE

Pour citer cet article :

LEBUNETEL J., FARNOLE P.. (2011). Impact de digues ISC sur la dynamique littorale : Etude de cas des plages de Saint-Hilaire de Riez en Vendée. Revue Paralia, Vol. 4, pp 9.1-9.10.

DOI:10.5150/revue-paralia.2011.009 (disponible en ligne - http://www.paralia.fr - available online) 


\section{Introduction}

\subsection{Description du littoral concerné}

Le domaine d'étude s'étend de la plage des Demoiselles à celle des Mouettes, plus au sud (voir figure 1). L'activité touristique, considérée comme un enjeu économique majeur, a nécessité des aménagements et des équipements dimensionnés pour répondre à ces besoins. Le littoral de Saint-Hilaire de Riez bénéficie de la protection naturelle de l'île d'Yeu qui fait écran principalement aux houles d'Ouest à Nord-Ouest et protège plus particulièrement le littoral Nord de Saint-Hilaire de Riez. Toutefois, pour préserver les installations en front de mer, des ouvrages de défense de type perré ont été mis en place pour stabiliser le trait de côte là où le recul du rivage est manifeste.

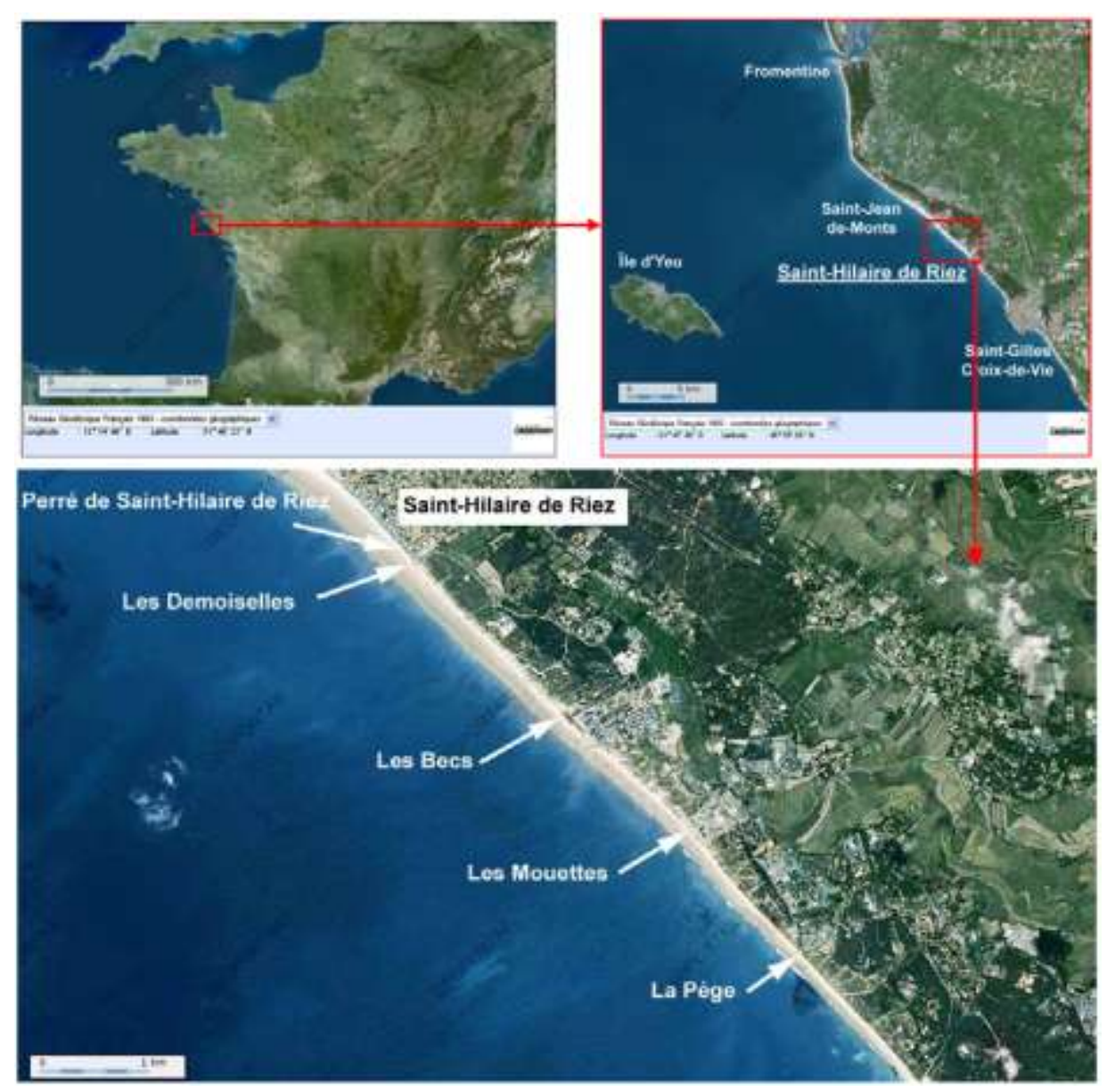

Figure 1. Localisation des sites d'étude sur le littoral de Saint-Hilaire-de-Riez.

Les principaux ouvrages de protection sur le littoral de Saint-Hilaire sont recensés ici :

a) Le perré maçonné de la plage des Demoiselles construit de 1974 à 1979, et renforcé par une butée de pied en enrochements en 1996 à son extrémité sud qui fait transition avec le cordon dunaire. 
Le mur maçonné présente très localement des signes de faiblesses au niveau du parement et des joints. Au sud du perré maçonné se situe un perré en enrochements prenant appui sur un remblai qui a été installé pour répondre à un problème d'érosion qui se manifeste généralement dans la zone de transition entre le mur de protection de la promenade et le cordon dunaire. Il sert aussi de rampe d'accès à la plage pour des engins. Il présente la particularité d'être proéminent vers la mer par rapport au mur de la promenade. Cette situation conduit à observer des formes d'érosion au niveau de la plage et de la dune attenante non protégées.

b)Les perrés en enrochements devant la plage des Becs et des Mouettes, mis en place, dans un premier temps, pour aménager des accès à la plage avant 1989 puis pour maintenir les terre-pleins aménagés en parking depuis 1998. La cale de mise à l'eau de la plage des Becs joue le rôle d'épi. Les perrés en enrochements de ces deux plages sont postés en avant sur l'estran et sont fragilisés de manière significative en particulier à leurs extrémités (voir figure 2).

c) Une quarantaine d'épis ont été construits entre 1968 et 1974 depuis le Nord du lotissement des Becs jusqu'au droit de la Parée Préneau (au sud de la Pège). Ces nombreux épis plus ou moins déstructurés semblent n'avoir aucun effet sur le transit sédimentaire (voir figure 3). Cependant, ils sont un indicateur du recul du trait de côte. Leur limite haute est en retrait de 25 à $50 \mathrm{~m}$ par rapport au cordon dunaire.

d) Les zones dunaires naturelles sont équipées de ganivelles. Elles sont installées sur une ligne en pied de talus pour éviter le piétinement par les estivants mais sont sans effet sur la protection du cordon dunaire.

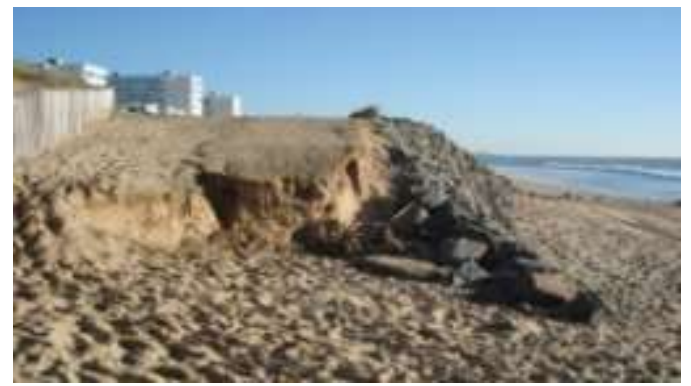

(a)

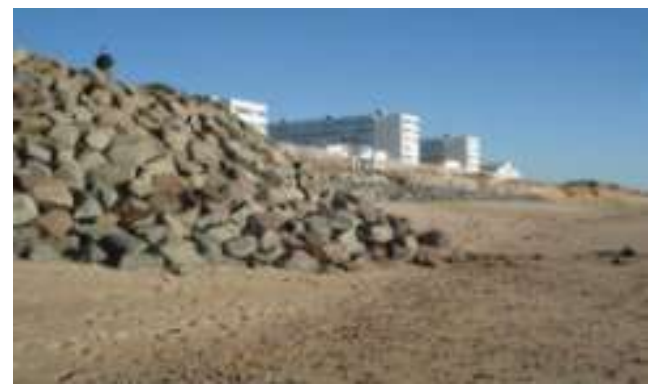

(b)

Figure 2. Site des Mouettes Nord du perré : (a) Érosion à l'extrémité;(b) Pied de talus.

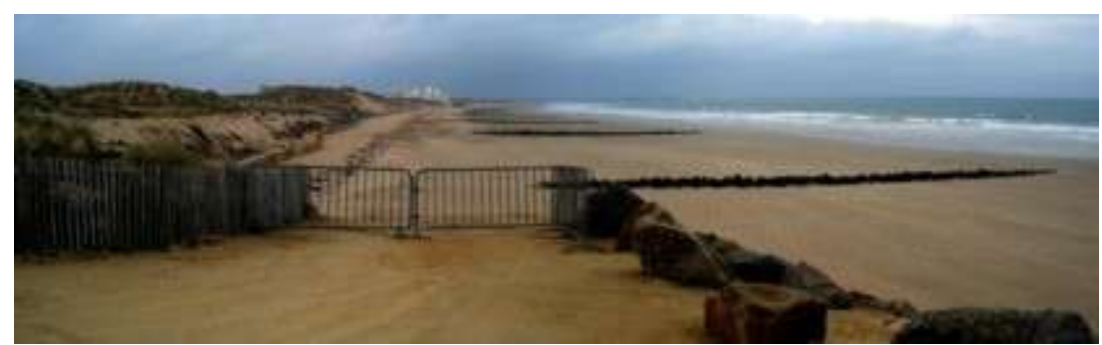

Figure 3. Batterie d'épis prise du haut du perré des Becs vers le sud. 


\subsection{Les digues classées}

Ces ouvrages classés ISC répondent à une réglementation particulière du fait de leur niveau de protection vis à vis des biens et des personnes contre les inondations. La mise en place de ce classement au titre de la sécurité civile est particulièrement suivie en Vendée (RAISON, 2008).

L'Etat effectue le recensement des digues de protection contre les inondations. Les digues sont classées en fonction de leurs caractéristiques (hauteur) et de la population protégée (classes A, B, C, D définies par décret du 11 décembre 2007). Selon chaque classe d'ouvrage, des prescriptions sont imposées (document de référence MISE 83).

L'Etat exerce le contrôle des digues intéressant la sécurité publique, au titre de ses pouvoirs de police de l'eau.

La gestion et l'entretien des digues relèvent de la compétence du maître d'ouvrage, qu'il s'agisse de propriétaires privés regroupés ou non en associations, de collectivités locales ou de l'Etat. Il est pleinement responsable, au niveau civil comme au niveau pénal des dommages qui peuvent être occasionnés par l'ouvrage et en particulier, le cas échéant, par sa rupture. En cas d'incident ou d'accident, sa responsabilité peut être engagée pour faute, négligence, imprudence, vice d'entretien ou défaut de construction (articles 1382 et suivants du code civil). Les modalités de mise en œuvre du contrôle des digues par l'Etat sont fixées par une circulaire. Ce contrôle consiste à vérifier que le maître d'ouvrage remplit ses obligations de maintien en bon état de sa digue et à contrôler qu'il effectue bien pour cela un suivi régulier et adéquat (consignes de surveillance et d'entretien, visites régulières et après un évènement météorologique sévère, ...).

Les digues ISC entrent dans la catégorie B pour les digues des Becs des Mouettes. Dans ce cadre, les obligations du maître d'ouvrage consistent à réaliser les dossiers suivants : un diagnostic de sûreté digue existante ; un dossier des ouvrages ; une visite technique approfondie tous les ans ; un rapport de surveillance périodique entre 1 et 5 ans ; un cahier de suivi des digues. L'étude de danger est aussi demandée pour évaluer l'aléa de submersion. En l'occurrence, pour une digue ISC en zone littorale l'appréciation de l'aléa de submersion s'appuie sur l'étude hydrosédimentaire.

C'est dans ce cadre de suivi des ouvrages que cette étude a été réalisée. Après diagnostic terrain, il apparaît que ces digues sont en mauvais état. Certes elles permettent une protection contre la mer mais leur position, particulièrement en avant sur l'estran, pose problème du fait du recul du trait de cote de part et d'autre de ces ouvrages. En effet, les accès à la mer, les avancées sur l'estran, conduisent à un durcissement du trait de côte. Ils ont un impact significatif sur les houles et engendrent des vitesses importantes à proximité. Ces ouvrages amplifient ainsi les phénomènes de transport sédimentaire. En conséquence de quoi les ouvrages se dégradent (affaissement des digues en enrochement, fragilisation par les fortes houles et courants, dégradation du géotextile...) et détériorent prématurément le littoral à proximité, avec des reculs prononcés du trait de cote particulièrement à proximité des zones aménagées. Il est donc 
nécessaire de repenser l'aménagement du littoral de Saint Hilaire afin de répondre à long terme à cette problématique. Notons que les résultats des auteurs sur l'impact des ouvrages sur l'évolution des fonds sont dispersés et ceci est du à la difficulté de compréhension des phénomènes et au nombre de paramètres à considérer. Cependant il a été montré que l'impact d'une digue frontale sur la bathymétrie environnante est négatif, remettant en cause la stabilité de l'ouvrage (SAMAT, 2007). Après cette première partie de présentation de l'étude, la chaîne de calcul utilisée pour modéliser l'état actuel et aménagé vis à vis des houles, courants et du transport sédimentaire associé est présentée dans la deuxième partie. Dans la troisième section sont présentés les résultats de ces simulations et la comparaison entre ceux issus de l'état aménagé et initial. Enfin, les conclusions seront émises dans la cinquième partie.

\section{Modélisation}

La chaîne de calcul mise en place est constituée de trois principales étapes réalisées à l'aide des logiciels REFONDE, REFLUX et REFLUX-SEDIM, développés intégralement par le CETMEF, qui modélisent respectivement l'agitation de la houle, les courants et le transport sédimentaire (http://www.cetmef.developpementdurable.gouv.fr/). Deux domaines ont été définis afin de couvrir au mieux les sites d'intérêt. Un maillage adaptatif a été réalisé pour obtenir des résultats précis à proximité des sites et limiter le nombre d'éléments et le temps de calcul.

\subsection{Modélisation de l'agitation}

Le logiciel REFONDE permet, à partir de données de houle et de bathymétrie actualisées, de réaliser la première étape de la chaine de calcul. Celle-ci consiste à reproduire un modèle d'agitation permettant d'étudier en détail les caractéristiques de la houle à la côte, considérant des houles irrégulières, et en particulier son incidence sur les ouvrages. Ce code aux éléments finis résout l'équation de Berkhoff prenant en compte les phénomènes de diffraction, réfraction et réflexion. Il permet de calculer la hauteur et la phase pour chaque train de houle.

\subsection{Modélisation des courants}

La deuxième étape de la chaîne de calcul consiste à mettre en œuvre la modélisation de la courantologie à l'aide du logiciel REFLUX. Celui-ci est tout particulièrement adapté aux problèmes de navigation, à la protection contre les inondations, à l'évaluation des conséquences de l'implantation d'ouvrages, en sites maritimes, fluviaux et estuariens. Ce code aux éléments finis résout les équations de St Venant et permet d'intégrer entre autres l'action de la marée et de la houle sur les courants via un couplage avec REFONDE (DEBAILLON et al., 2001). Un mode de résolution instationnaire est donc choisi avec une méthode de calcul de Newton-Raphson avec un modèle de bancs couvrants/découvrants. En nature, la frontière du domaine mouillé peut varier au cours 
du temps, particulièrement en cas de fort marnage. Ceci est intégré dans le modèle bancs couvrants/découvrants, qui permet de détecter la frontière de la zone mouillée et de résoudre sur les éléments partiellement mouillés les équations de Saint-Venant modifiées.

\subsection{Modélisation du transport sédimentaire}

Le dernier aspect de la modélisation concerne l'estimation du transport sédimentaire. REFLUX-SEDIM calcule l'évolution des fonds et le transport solide à partir des équations de conservation de la masse et de transport par charriage et suspension (Loi de Van Rijn). A l'aide d'un couplage avec le module hydrodynamique REFLUX, il est possible d'évaluer les zones de potentiel dépôt ou érosion sur site après plusieurs cycles de marée.

\section{Résultats et aménagements proposés}

\subsection{Dérive littorale}

Les caractéristiques de houle considérées sont représentatives d'une houle annuelle et obtenues à partir de la base de données in situ ANEMOC. Le logiciel REFONDE appliqué à notre domaine d'étude a permis de montrer qu'au nord du secteur d'étude, l'obliquité, à l'origine de la dérive littorale, est faible (< à $3^{\circ}$, voir figure $\left.4 \mathrm{a}\right)$. Ceci permet de conclure à un faible transit sédimentaire le long de la côte légèrement dirigé vers le nord. La présence d'ouvrages de protection fige l'orientation de la côte et participe à la déstabilisation de la dynamique littorale, particulièrement en cas d'obliquité forte comme mesurée dans le secteur sud du domaine étudié (de $-1,2^{\circ}$ à $-9^{\circ}$, voir figure 4b), indiquant cette fois un transit important dirigé vers le sud.

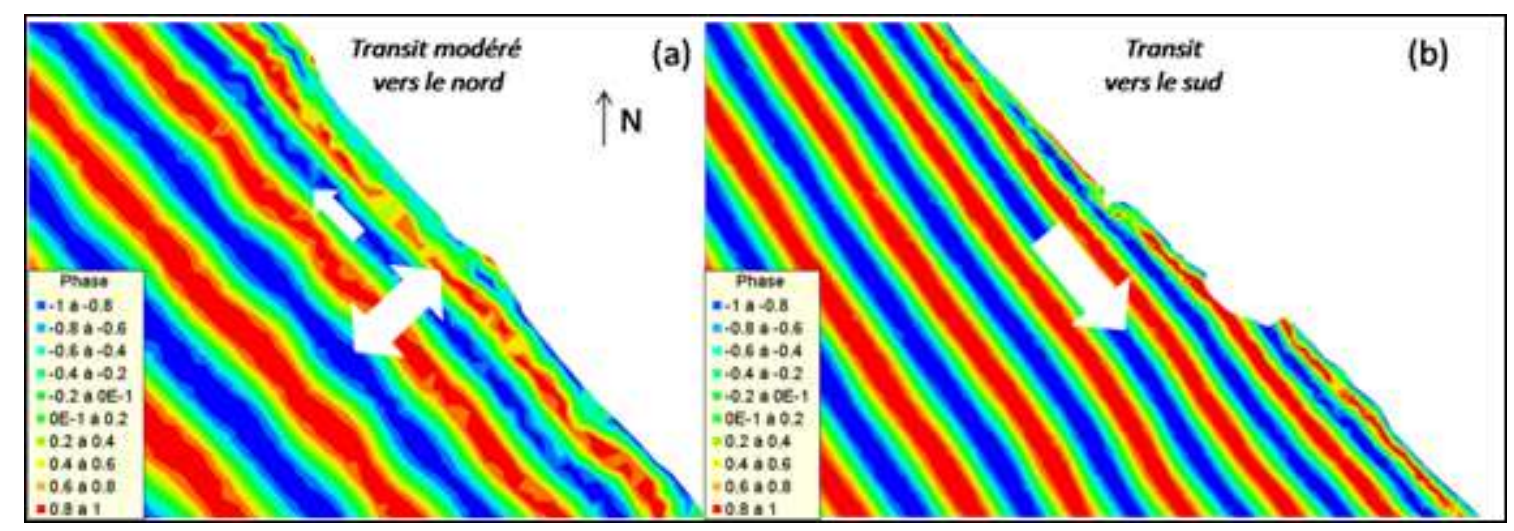

Figure 4. Phases de houle résultantes du modèle d'agitation à l'approche pour une houle annuelle $\left(T_{p}=12 \mathrm{~s} ; H_{s}=1,4 \mathrm{~m}(\grave{a}-5 \mathrm{mCM}) ; \theta_{p b}=235^{\circ} ; \theta_{p a}=231^{\circ}\right):(a)$ des

Demoiselles ; $(b)$ des Becs. 
L'agitation, ayant un impact significatif sur le transport sédimentaire notamment à proximité des ouvrages (BRIERE, 2006), a fait l'objet d'un examen particulier.

Plusieurs secteurs de houle ont été considérés pour les modélisations, directions dominantes sur le secteur d'étude. Les simulations de l'agitation pour les conditions de houle annuelles ont montré, que dans l'état actuel, les hauteurs de houle résiduelle à la côte sont relativement importantes, de l'ordre de 1,5 m à 2,1 m. Un exemple en est présenté sur la figure 5a, à proximité de la plage des Becs. Ces conditions d'agitation relativement élevées couplées à l'obliquité de la houle et aux variations d'orientation des ouvrages par rapport au trait de côte induisent un transit sédimentaire relativement important.

Les aménagements proposés sont les suivants :

- Réhabilitation des digues ISC sur les plages des Becs et des Mouettes, avec réalignement sur la ligne du rivage qui présente une dissymétrie entre nord et sud ;

- Rechargements massifs de plages sur 1100 m entre les Becs et les Mouettes ;

- Suppression de la digue des Demoiselles, proéminente par rapport au trait de côte, afin de réhabiliter le cordon dunaire.

La suppression des avancées sur l'estran permet de laisser le trait de côte évoluer afin de rétablir une dynamique stable sur ce littoral. Les aménagements prévus permettent de réduire l'effet de réflexion néfaste des ouvrages (voir figure $5 \mathrm{~b}$ ). Cette réduction de l'agitation est de l'ordre de $25 \%$ environ au niveau des ouvrages.

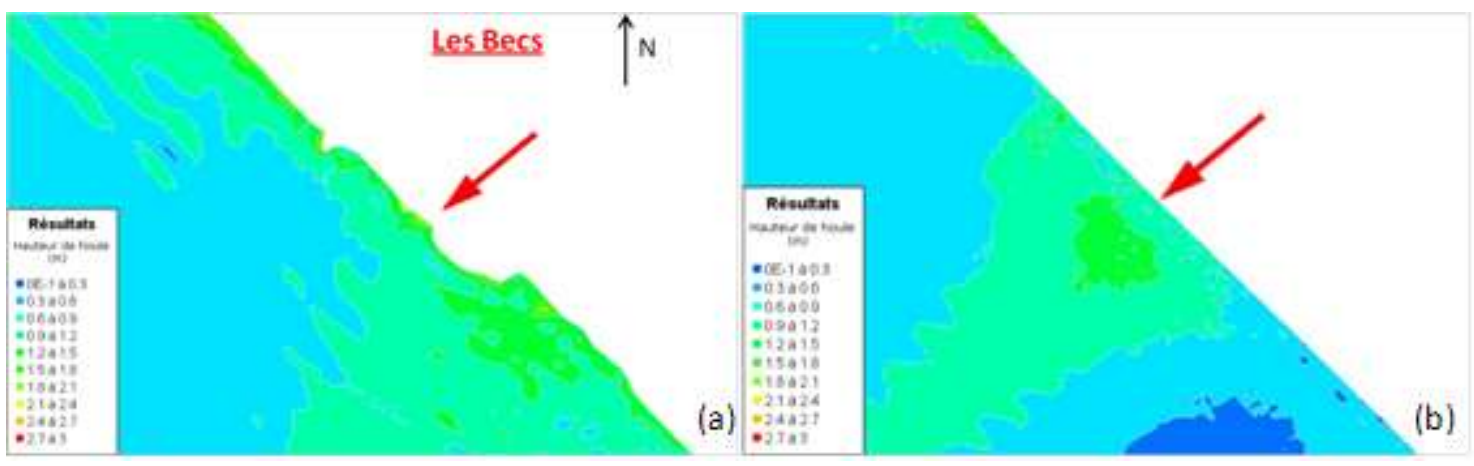

Figure 5. Hauteurs de houle à la côte (Conditions au large : $T_{p}=12 \mathrm{~s} ; H_{s}=1,4$ $\left.\theta_{p}=232^{\circ} \mathrm{N}\right):(a)$ état initial ; $(b)$ état aménagé.

\subsection{Impacts des ouvrages sur les courants et le transport sédimentaire}

Les résultats du modèle de courantologie montrent l'impact significatif des ouvrages de protection contre la mer en enrochements des plages des Becs, des Mouettes et des Demoiselles sur les courants engendrés par l'action simultanée de la houle et de la marée. En effet, ces ouvrages constituent un décrochement par rapport au trait de côte vers l'estran en conséquence de quoi les courants sont très perturbés à proximité de ceux-ci. Les forts courants locaux et les mouvements tourbillonnaires générés (voir 
figure 6a) indiquent un risque important non seulement de déstabilisation du pied de digue mais aussi d'érosion du haut de plage en aval.

Dans cette région sont préconisés un recul des ouvrages et un rechargement massif en sable. Les courants générés dans une telle situation sont moindres (voir figure $6 \mathrm{~b}$ ) ce qui laisse présager d'un transport moindre (voir figure 7) et d'une meilleure stabilité des ouvrages. En effet, il en résulte un meilleur amortissement des houles, les recirculations et accélérations observées dans l'état initial disparaissent et la plage retrouve un profil d'équilibre. La réduction des vitesses est d'environ 20\% pour le site des Demoiselles et peut aller jusqu'à $70 \%$ sur le site des Becs et Mouettes.

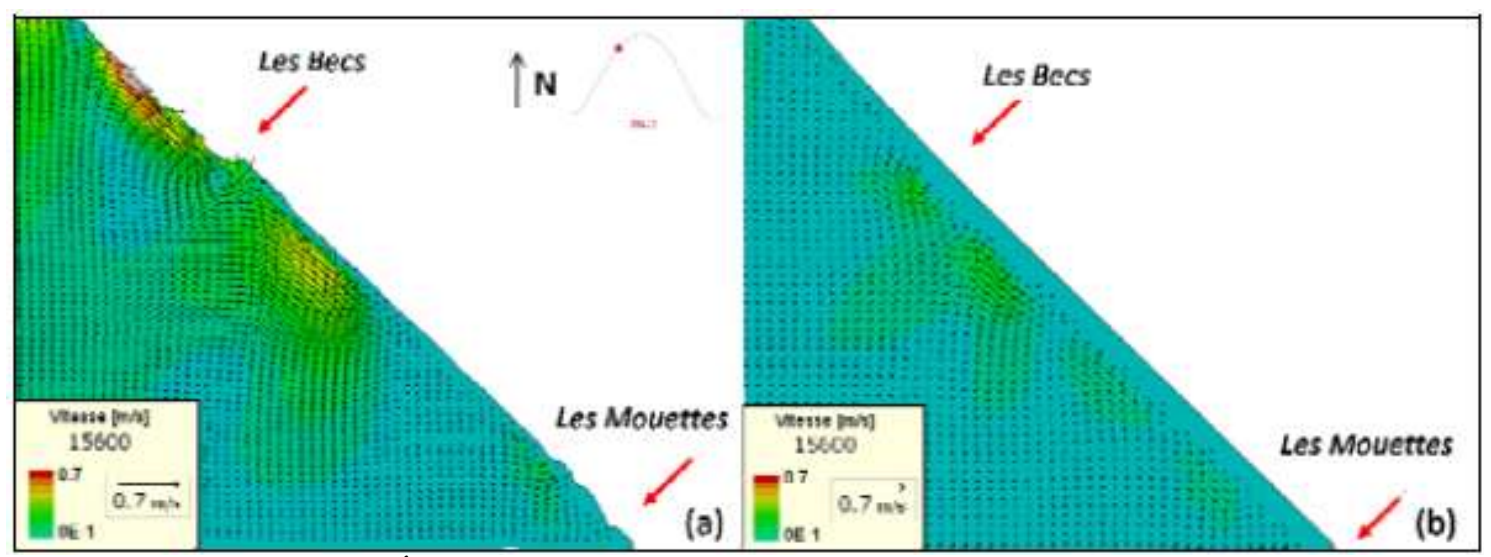

Figure 6. État des courants : (a) état initial ; (b) état aménagé.

La modélisation via REFLUX-SEDIM a permis d'estimer les zones de potentiel dépôt/érosion au cours du temps. On détecte l'affouillement effectif au pied des ouvrages et les zones de déstabilisation sédimentaire sur l'estran dans le cas de la situation actuelle. A l'aide de formules empiriques classiques faisant intervenir notamment l'obliquité de la houle, il est possible d'estimer un débit solide. Parmi ces formules, nous pouvons citer celle de Kamphuis (KAMPHUIS, 1991) :

$$
Q=2.27 H_{s b}^{2} T_{p}^{1.5} \tan \beta^{0.75} d_{50}^{-0.25}\left(\sin 2 \alpha_{b}\right)^{0.6}
$$

Avec : $Q\left[\mathrm{~m}^{3} / \mathrm{s}\right]$ : débit solide $H_{s b}[\mathrm{~m}]$ : hauteur de houle au déferlement ; $T_{p}[\mathrm{~s}]$ : période pic de la houle $; \tan (\beta)$ : pente de la plage $; d_{50}[\mathrm{~m}]$ : diamètre médian des sédiments ; $\alpha_{b}$ : obliquité de la houle au déferlement.

Une autre formulation largement utilisée est celle du CERC (CERC, 1984) :

$$
Q=K \frac{\rho \sqrt{g}}{16 \sqrt{\gamma}\left(\rho_{s}-\rho\right)(1-n)} H_{s b}^{5 / 2} \sin \left(2 \alpha_{b}\right)
$$

Avec $K$ : coefficient empirique ( $K=0,39$ CERC, $1984 ; K=f\left(d_{50}\right)$ DEL VALLE et al., $1993) ; n=0,4$ : porosité du sable; $\rho$ et $\rho_{s}\left[\mathrm{~kg} / \mathrm{m}^{3}\right]$ : masse volumique de l'eau et du sédiment ; $\gamma$ : indice de déferlement. 
La dispersion des résultats obtenus à partir des différentes formulations selon le site d'étude et les conditions hydrodynamiques peut être importante. Les coefficients empiriques sont très variables selon les auteurs (ROSAT et al., 2002), ce sont donc des informations à prendre avec précautions. Les calculs sont réalisés pour l'état initial et la solution aménagée permettant d'estimer un écart de transit entre les deux applications.

A partir de ces calculs, nous pouvons estimer que l'aménagement envisagé permet de réduire de $20 \%$ environ le transit sédimentaire au droit de la plage des Becs. L'incidence de l'aménagement sur le transport sédimentaire contribue à l'amélioration de la stabilité de la plage dans le secteur de la plage des Becs.

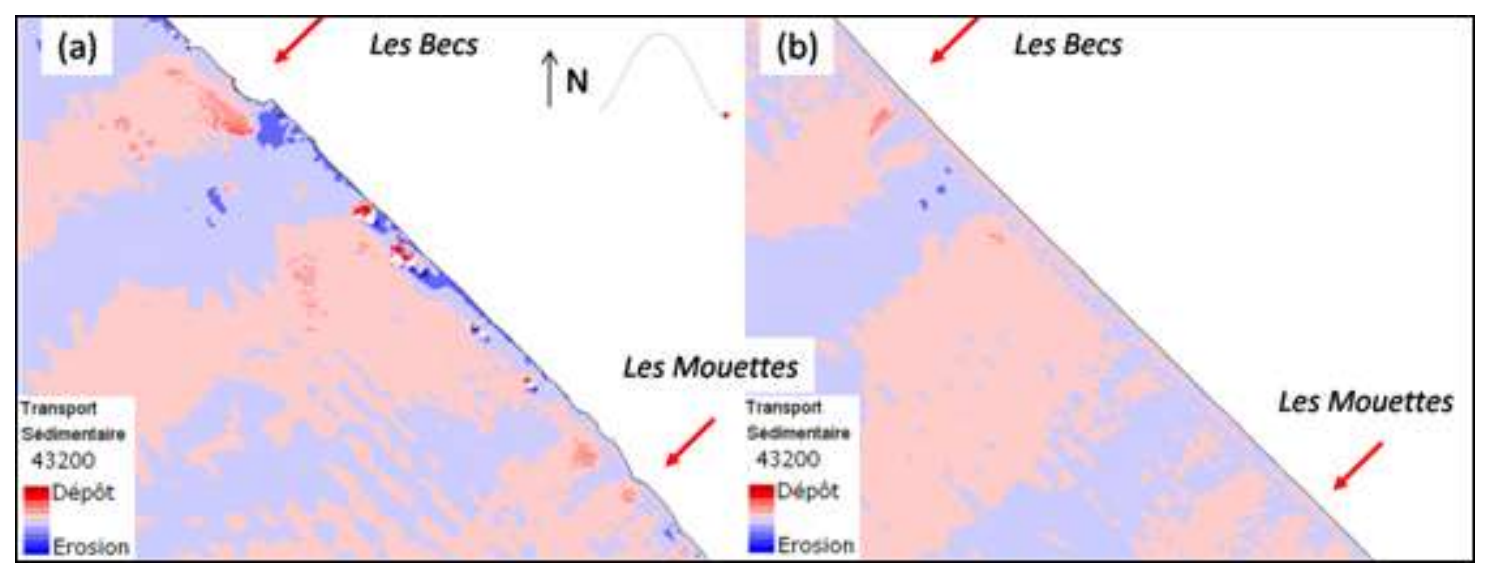

Figure 7. Estimation du transport sédimentaire: (a) état initial ; (b) état aménagé.

\section{Conclusions}

La modélisation numérique de l'état aménagé montre une nette amélioration de la situation en termes de courants et de transport sédimentaire. De plus, la faible agitation générée par les ouvrages sera réduite en conséquence des rechargements de plage préconisés. Bien que l'observation et l'expertise soient indispensables pour la bonne réussite d'un projet comme celui-ci, la modélisation est un outil précieux qui permet, sous réserve d'avoir des mesures in situ de bonne qualité et actualisées, de quantifier les améliorations afférant aux nouveaux aménagements et d'en apporter un support visuel. Les digues ISC des Mouettes et des Becs nécessitent une complète réhabilitation pour sécuriser les installations urbaines. De même, la sécurité du camping situé au sud de la Pège n'est plus assurée du fait du recul du trait de cote. L'emplacement du camping va être modifié afin que sa frontière, dorénavant alignée avec le trait de cote, se situe à $100 \mathrm{~m}$ de celui-ci. La réhabilitation des digues ISC accompagnée d'un rechargement massif en sable aura pour effet de réduire à un niveau faible l'aléa de submersion.

La stratégie adoptée de recul du trait de cote a le mérite d'être peu intrusive pour le littoral mais surtout permet de rétablir un profil stable et ainsi de sécuriser de manière durable les biens et les personnes situées en amont. Cette stratégie fait partie des prescriptions du Grenelle de la mer qui prévoit, au sein de l'engagement 74 intitulé : 
"Anticiper et prévenir les risques naturels et technologiques", de "développer une méthodologie et une stratégie nationale (collectivités et Etat) pour la gestion du trait de côte, pour le recul stratégique et la défense contre la mer".

\section{Références bibliographiques}

BRIERE C. (2005). Etude de l'hydrodynamique d'une zone côtière anthropisée l'embouchure de l'Adour et les plages adjacentes d'Anglet. Thèse, Université de Pau et des Pays de l'Adour, p. 141.

CERC -Coastal Engineering Research Centre- (1984). Shore Protection Manual, volumes I \& II. Department of the Army, US Army Corps of Engineers.

DEBAILLON D., SERGENT P. et ZHANG B., (2001), Morphological evolution behind a detached shore-parallel breakwater, Coastal Dynamics, pp 46-54, Lund (Suède).

DEL VALLE R., MEDINA R., LOSADA M. A. (1993). Dependence of Coefficient K on Grain Size. Technical Note ${ }^{\circ} 3062$ Journal of Waterway, Port, Coastal and Ocean Engineering, Vol 119, $\mathrm{n}^{\circ} 5$ September/October, pp 568-574.

KAMPHUIS J.W. (1991). Alongshore sediment transport rate. Journal of Waterways, Port, Coastal and Ocean Engineering ASCE, 117(6), pp 624-641. doi:10.1061/(ASCE)0733950X(1991)117:6(624)

RAISON S. (2008). Le classement des digues littorales au titre de la sécurité civile : un exemple de mise en ouvre en Vendée. $\mathrm{X}^{\text {èmes }}$ Journées Nationales Génie Côtier - Génie Civil, Sophia Antipolis, pp 283-292. doi:10.5150/jngcgc.2008.027-R

ROSAT J., WALTON T., BODGE K. (2002). Coastal Engineering Manual Chapter III 2 Longshore sediment transport.

SAMAT O. (2007). Efficacité et impact des ouvrages en enrochement sur les plages microtidales. Le cas du Languedoc et du Delta du Rhône. Thèse, Université AixMarseille III, p.341. 